\title{
Early Diagnosis and Treatment of Coronary Heart Disease in Symptomatic Subjects With Advanced Vascular Atherosclerosis of the Carotid Artery (Type III and IV b Findings Using Ultrasound)
}

\author{
Ansgar Adams $^{\mathrm{a}, \mathrm{d}}$, Waldemar Bojara ${ }^{\mathrm{b}}$, Klaus Schunk ${ }^{\mathrm{c}}$
}

\begin{abstract}
Background: A study was conducted as to whether the early diagnosis of coronary heart disease (CHD) in symptomatic patients with advanced atherosclerosis of the carotid artery was more successful using ultrasound technology than exercise electrocardiography (ECG).

Methods: Within the scope of an occupational screening program using subjects from diverse employment sectors, people were given the opportunity to determine their risk of heart attack. During the study, the total plaque area (TPA), the maximum plaque thickness in the carotid artery and the PROCAM scores of 3,513 healthy men and 2,088 healthy women between the ages of 20 and 65 were determined. During the subsequent follow-up study, 36 subjects developed symptoms such as exertional dyspnea, atypical angina pectoris (AP) or typical AP. Four patients displayed no symptoms. The initial cardiac diagnostic testing was conducted on 31 patients using an exercise ECG, four patients were assessed using a coronary angiogram, and five further patients were assessed using a computed tomography (CT) coronary angiogram. An ultrasound examination of the carotid artery of 39 patients revealed a type IV b finding and in one patient, the examination revealed a type III finding.
\end{abstract}

Results: In 17 patients, the PROCAM score was $<10 \%, 13$ patients had a score of $10-20 \%$ and 10 patients had a score of $>20 \%$. In the final analysis, only two patients had entirely smooth coronary arteries, seven had coronary sclerosis, seven had a $30 \%$ stenosis, one had a $30-40 \%$ stenosis, one had a $40 \%$ stenosis, and 22 patients had a stenosis $\geq 50 \%$, and in extreme cases, a left main coronary artery stenosis with three-vessel disease was shown. The exercise ECG only achieved a true positive result in four patients, and in 21 patients, the

\footnotetext{
Manuscript accepted for publication January 09, 2017

${ }^{a} B \cdot A \cdot D$ Health Care and Safety Technology Centre GmbH, Koblenz, Germany ${ }^{b}$ Community Clinic Mittelrhein, Kemperhof II, The Cardiology Clinic, Koblenz, Germany

${ }^{\mathrm{c} C o m m u n i t y ~ C l i n i c ~ M i t t e l r h e i n, ~ K e m p e r h o f ~ C l i n i c ~ f o r ~ D i a g n o s t i c s ~ a n d ~ I n t e r-~}$ vention in Radiology, Germany

${ }^{\mathrm{d} C}$ Corresponding Author: Ansgar Adams, B·A·D Health Care and Safety Technology Centre GmbH, Koblenz, Germany.

Email: ansgar.adams@bad-gmbh.de
}

doi: https://doi.org/10.14740/cr516w result was false negative.

Conclusions: Symptomatic patients with advanced atherosclerosis of the carotid artery (type III and type IV b findings) had a high risk for CHD. The diagnosis of CHD is better achieved by using carotid duplex than with an exercise ECG. Early treatment of the disease improves the patient's prognosis.

Keywords: Total plaque area; Carotid duplex; Cardiovascular risk; Coronary heart disease; PROCAM score

\section{Introduction}

Within the scope of an occupational screening program in the Koblenz area, people working in diverse sectors of the economy such as chemicals, glass, pharmaceuticals, administration, steel production, social services, paper production, printing, ceramics, IT, university, universities of applied science and retail were given the opportunity to determine their risk of heart attack. During the study, the total plaque area (TPA), the maximum plaque thickness in the carotid artery and the PROCAM scores of 5,601 healthy subjects (who had had no previous history of a cardiovascular event) between the ages of 20 and 65 (3,513 men and 2,088 women) were determined.

In the mean follow-up study of period of $35.2 \pm 21$ months, seven strokes and 20 heart attacks have occurred to date. Seven bypass operations and four stent implantations have been performed, and stenoses (70\% and 90\%, respectively) have been revealed in two patients who underwent a coronary angiogram. Four subjects exhibited a type III finding and 26 subjects showed a type IV $b$ finding in the ultrasound examination of the baseline study.

The testing method involving the measurement of plaque burden and maximum plaque thickness was validated by means of a blinded examination of 500 patients in hospital 1 day prior to a scheduled coronary angiogram. This allowed the classification of the ultrasound findings into four types [1]. Patients with a type III or a type IV b finding in the ultrasound examination revealed one-, two- or three-vessel disease in $83 \%$ of cases. Eighty-seven percent of the patients suffering from a stenosing coronary heart condition were accurately predicted 


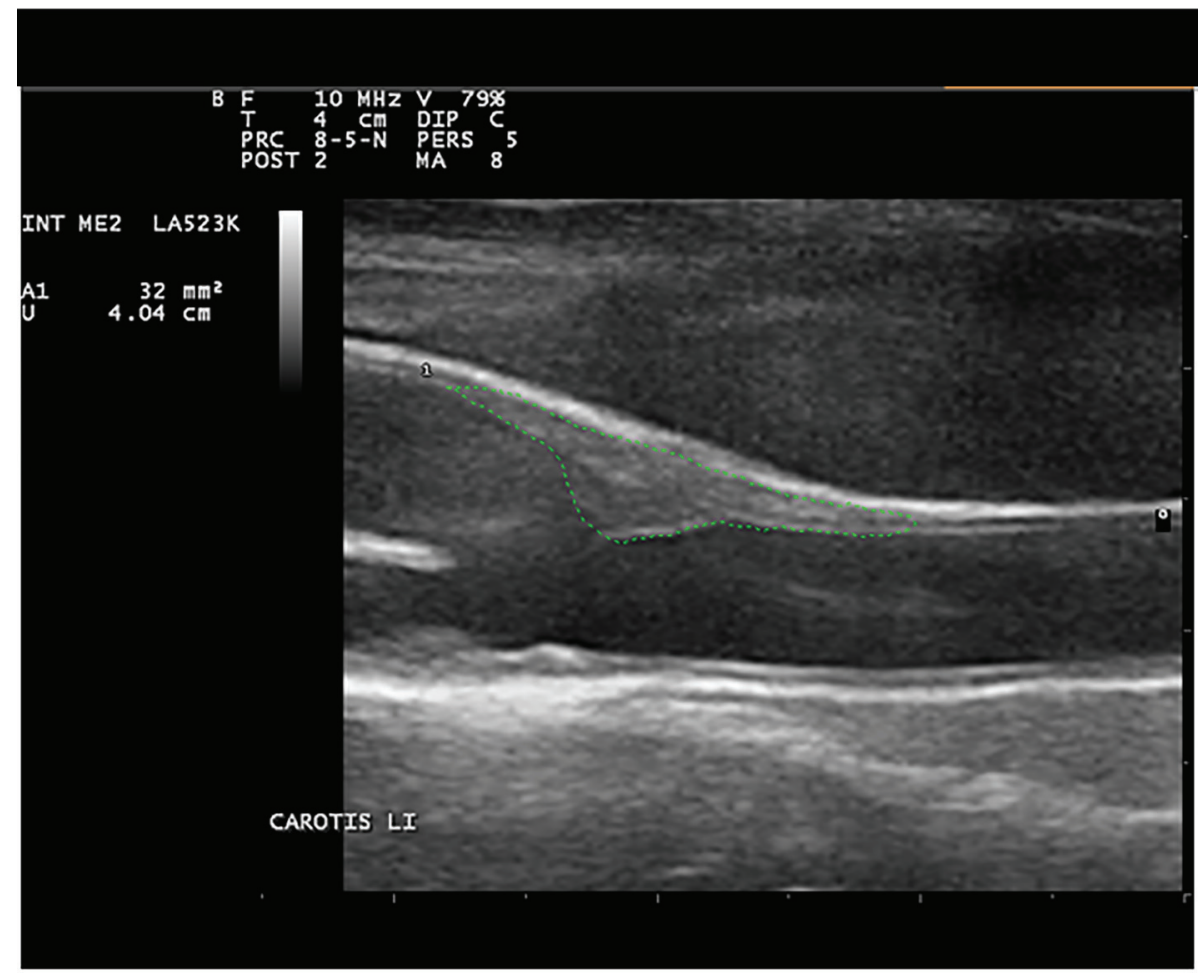

Figure 1. Type III in the carotid duplex with TPA under the age-related cut-off.

(type III or type IV b finding during the carotid duplex).

This means that subjects not yet displaying any symptoms of coronary heart disease (CHD) predominantly have advanced atherosclerosis of the carotid artery. The positive predictive value (PPV) for hospitalized patients is of course not transferable due to the much lower prevalence of atherosclerosis of the carotid artery in healthy subjects; however, the sensitivity of the carotid duplex in predicting CHD is not dependent upon its prevalence. Subsequently, 408 healthy subjects (73 of them women) with a type III or type IV b finding were contacted and specifically asked if they had experienced, or were experiencing symptoms such as exertional dyspnea or AP. Thirty-six subjects ( 30 men and six women aged between 43 and 61 years) complained of exertional dyspnea or of atypical AP or typical AP. Four subjects (three men and one woman) had no symptoms at all; however, these subjects elected to undergo further cardiac examinations. In all subjects, there was advanced atherosclerosis of the carotid artery (type III or type IV $b$ finding, Figs. 1 and 2). In 36 subjects, further cardiac assessment was recommended. A comparison was drawn between the results of the ultrasound examination and the exercise electrocardiography (ECG).

\section{Methods}

The carotid artery (common carotid artery both externally and internally using B-mode ultrasound) was examined on both sides, as far as could be made visible, for plaques on the long and short axis from the clavicle through to the temporo- mandibular joint in a caudal to cranial direction by means of anterior, anterolateral, lateral and posterolateral insonation. A portable ultrasound device with a $10 \mathrm{MHz}$ linear transducer of the type Imagic Agile and manufactured by the company Kontron Medical was used during testing. Only carotid artery wall thickness increases with an intima media thickness (IMT) $>1 \mathrm{~mm}$ were evaluated as plaque. Using the device's surface area measurement program, it was possible to identify all the plaques in a longitudinal section (the sum total of all plaque surface areas TPA) by tracing the perimeter of each plaque with the cursor at its maximum enlargement in the B-mode image. Furthermore, the maximum plaque thickness was measured. The findings of the ultrasound examinations were classified according to four types ranging from type I to type VI b [1]: type I: no or only minimal atherosclerosis of the carotid artery with a TPA of up to $24 \mathrm{~mm}^{2}$; type II a: only flat plaques $\leq 2 \mathrm{~mm}$ IMT with plaque surface area under age-related cut-off in the carotid artery; type II b: only flat plaques $\leq 2 \mathrm{~mm}$ IMT with a large plaque surface area above age-related cut-off in the carotid artery; type III: carotid plaques with an IMT $\geq 3.5$ $\mathrm{mm}$ and a plaque surface area under age-related cut-off; and type IV a: mixed type (with flat carotid plaques and plaques $>$ $2 \mathrm{~mm}$ IMT and a plaque surface area under age-related cut-off; type IV $b$ with flat carotid plaques and plaques $>2 \mathrm{~mm}$ IMT and a large plaque surface area over age-related cut-off).

The TPA cut-off in the subjects $<40$ years of age was 60 $\mathrm{mm}^{2}$, from 40 to 49 years of age, it was $80 \mathrm{~mm}^{2}$, from 50 to 59 years of age, it was $110 \mathrm{~mm}^{2}$, and from 60 to 65 years of age, it was $130 \mathrm{~mm}^{2}$. The cut-off for maximum plaque thickness was $>2 \mathrm{~mm}$, or irrespective of the TPA, it was $\geq 3.5 \mathrm{~mm}$. 


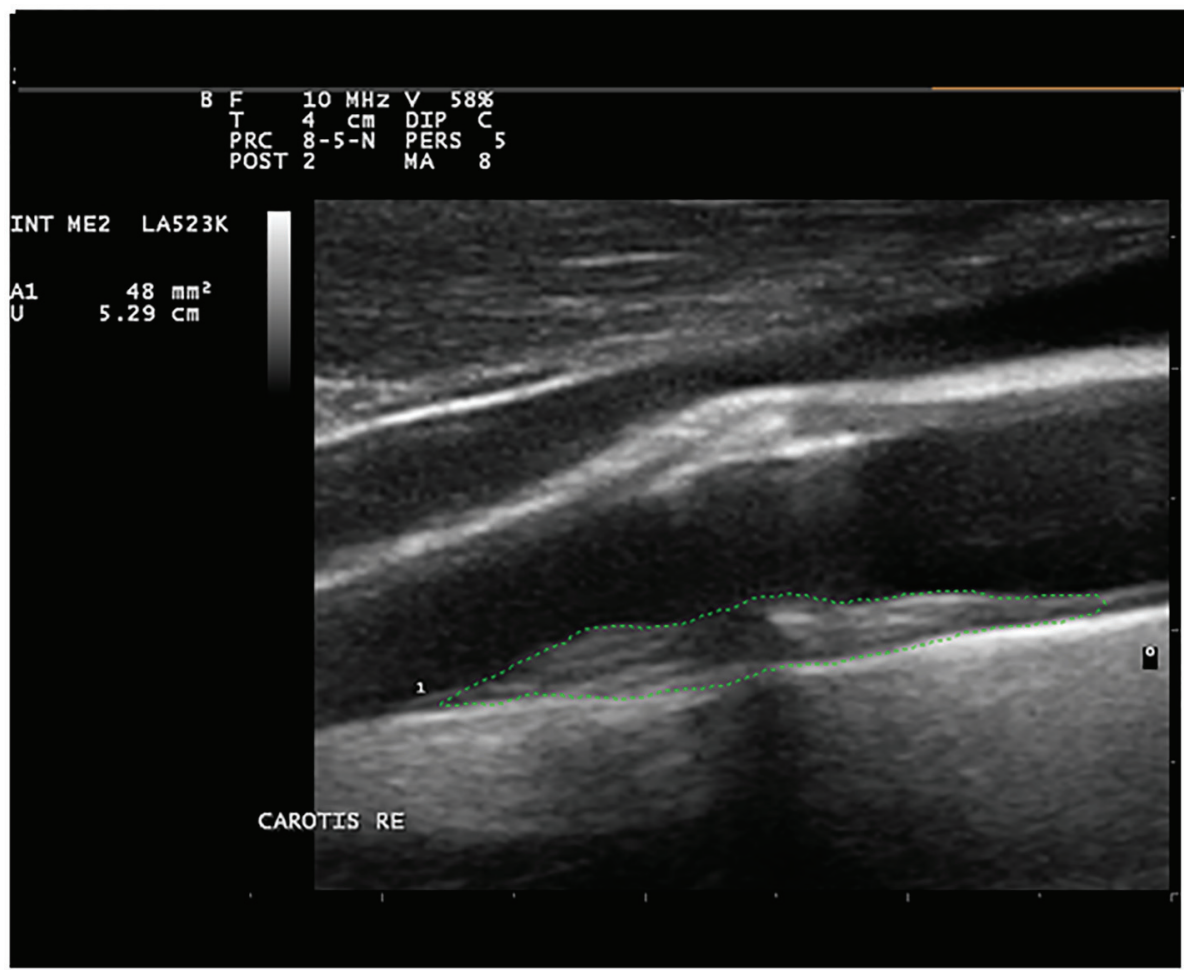

Figure 2. Type IV b in the carotid duplex with TPA above the age-related cut-off.

According to this classification, it was possible to predict coronary stenosis in patients who were examined 1 day prior to their undergoing a coronary angiogram with a sensitivity level of $87 \%$ [1].

The cardiac assessment was conducted using exercise ECGs, CT coronary angiograms and coronary angiograms.

The risk of heart attack was determined using the PROCAM score (www.assmann-stiftung.de).

\section{Results}

The initial cardiac diagnostic testing was conducted on 31 patients using an exercise ECG, four patients were assessed using a coronary angiogram, and five further patients were assessed using a CT coronary angiogram. An ultrasound examination of the carotid artery of 39 patients revealed a type IV b finding, and in one patient, the examination revealed a type III finding. The PROCAM score of 17 patients was $<10 \%, 13$ patients had a score between $10 \%$ and $20 \%$, and 10 patients had a score $>20 \%$. In the final analysis, two patients had entirely smooth coronary arteries, seven had coronary sclerosis, seven had a $30 \%$ stenosis, one patient had a $30-40 \%$ stenosis, one patient had a $40 \%$ stenosis, five patients had a $50 \%$ stenosis, two patients had a $60 \%$ stenosis, two patients had a $60-70 \%$ stenosis, two patients had a 70\% stenosis (a stent was inserted into one of these patients), four patients had an $80 \%$ stenosis (a stent was inserted into two of these patients), one patient had a $90 \%$ stenosis, two patients had a 95\% stenosis (stents were inserted into both these patients), two patients had a vascular occlusion (stents were inserted into both these patients), and two patients underwent emergency bypass surgery following a coronary angiogram (both patients had main stem stenosis, three-vessel disease). The exercise ECG only achieved a true positive result in four patients, and in 21 patients, the result was false negative. In the analysis, the patients with coronary sclerosis are not classified as sick. Among the 10 patients with a high PROCAM score, four had coronary sclerosis and six had a stenosis. Table 1 gives an overview of the clinical data. A type III or type VI b finding is present in $7.28 \%$ of the 5,601 subjects who were examined.

\section{Discussion}

Atherosclerosis is an inflammatory disease of the arteries and continues to be the most common cause of death (over $40 \%$ ) in the developed industrial nations. The disease can even begin from the age of 20 and often remains undetected until it is in its later stages, resulting in clinical events such as acute coronary syndrome (ACS), stable angina, apoplexy and peripheral artery disease (PAD). Several studies demonstrate that even insignificant coronary stenoses are associated with an increased risk of heart attack [2-4]. An extensive multicenter research study (CONFIRM study) using 27,000 CT coronary angiograms revealed that a statin therapy contributes towards an improvement in survival chances even in the case of stenoses $<50 \%$ [5]. The improvement in survival chances achieved by using statin therapy in the group of subjects with non-obstructive coronary heart disease was at a hazard ratio of $0.39,95 \%$ confidence 


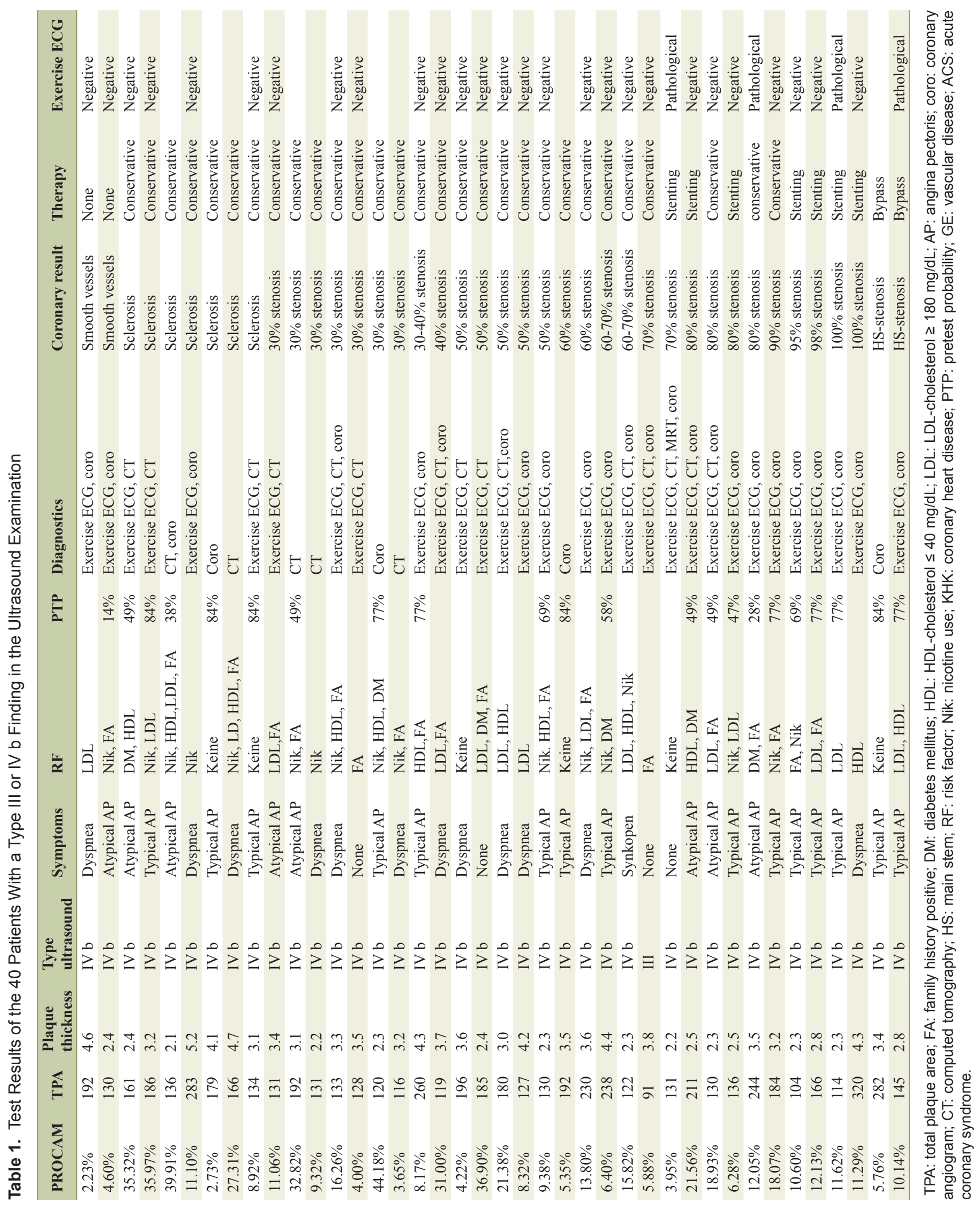


interval of $0.23-0.65$ and a $\mathrm{P}$ value of $<0.001$. This effect was not verifiable in the group with normal coronary arteries. It therefore appears important that atherosclerosis is both diagnosed and treated in its early stages in order to delay the final outcome of the disease as long into the future as is possible. In the case of out-patient diagnostics, the exercise ECG still plays an important role in the diagnosis of suspected CHD. The sensitivity of the exercise ECG in diagnosing coronary stenosis is only $45-50 \%$. The importance of the exercise ECG in diagnosing coronary stenosis was therefore downgraded in the most recent guidelines [6]. In the case of a pretest probability (PTP) of between $15 \%$ and $65 \%$, an exercise ECG is initially recommended. The exercise ECG only achieved a true positive result in four patients, and in 21 patients, the result was false negative; therefore no treatment was initiated with these patients. In view of the low number of subjects, it is not possible to evaluate these statistics using the PPV); however, the results indicate that the ultrasound examination is significantly more effective in the diagnosis of suspected CHD. It would be therefore desirable if a widely available, inexpensive, non-invasive diagnostic tool with a high level of accuracy and free from side effects was developed. The results of the study indicate that this could be achieved with the combination of TPA measurement and determining the maximum plaque thickness of the carotid artery. An evaluation of 500 ultrasound examinations carried out on patients 1 day prior to their undergoing a coronary angiogram revealed an $87 \%$ level of sensitivity in predicting a coronary stenosis of at least $50 \%$ following a type III or IV b ultrasound finding [1]. A further study which was conducted revealed similar results [7]. The follow-up study to date has demonstrated that subjects with advanced atherosclerosis (type III or type IV $\mathrm{b}$ findings) are at significantly increased risk of having cardiovascular disease. In the case of 40 patients (heart attack, stroke, bypass surgery, stent implantation, and existing stenosing coronary heart condition), 30 had a type III or a type IV b finding during the carotid duplex in the baseline study. From a further 40 subjects (36 with symptoms and four asymptomatic) with advanced atherosclerosis of the carotid artery who underwent cardiac diagnostic testing, 22 were found to have a stenosing coronary heart condition, nine had a stenosis of up to $40 \%$, and seven had coronary sclerosis. Only two patients had entirely smooth coronary arteries. Cardiac assessment using an exercise ECG is insufficient. In order to confidently rule out CHD, it is necessary to conduct a coronary $\mathrm{CT}$ even if tests for ischemia are negative. Determining the PROCAM score is meaningful since the notorious risk factors (RFs) are taken into consideration; however, the PROCAM score does not accurately display the risks associated with atherosclerosis of the carotid artery. Out of a total of 5,601 examined subjects, $7.28 \%$ had a type III or type IV b finding in the ultrasound examination. Forty-seven percent of the men examined with a type III or type IV b finding had a low PROCAM score, and in the cases of women, even $85 \%$ with a type III or type IV b finding had a low PROCAM score. From the 71 patients in total, $38.1 \%$ had a low PROCAM score and $36.6 \%$ had a score indicating intermediate risk. This demonstrates that the PROCAM score is of little preventative value in individual cases. Using ultrasound screening of the carotid artery on subjects with a high level of cardiovascular risk means that they can be both easily and inexpensively diagnosed and thus treated early. It is very probable that a number of asymptomatic subjects with advanced atherosclerosis (particularly those subjects who also have several other RFs for cardiovascular disease) already have an existing asymptomatic coronary heart condition which would respond favorably to treatment with statins. Further research is therefore required in this area in order to promote better preventative medicine.

\section{Conclusions}

Subjects with symptoms such as exertional dyspnea, atypical or typical AP, and advanced atherosclerosis of the carotid artery (type III or type IV b finding) have a high risk of CHD. Cardiac assessment using an exercise ECG is insufficient since the sensitivity level of this test is too low. An early diagnosis of $\mathrm{CHD}$ using ischemia imaging proves unsuccessful since no ischemia is present. The PROCAM score also does not close this diagnostic gap since it does not accurately display the risks associated with atherosclerosis. Further clarification is required as to whether (also in the case of asymptomatic subjects with advanced atherosclerosis and several other RFs) a coronary heart condition is already present that would respond favorably to treatment with statins.

\section{Limitations}

Only a small number of patients were examined.

\section{Conflicts of Interest}

The authors confirm that they have no conflicts of interest.

\section{References}

1. Adams A, Bojara W. Prediction of coronary artery stenosis by measurement of total plaque area and thickness versus intima media thickness of the carotid artery. Herz. 2015;40(5):817-822.

2. Proudfit WL, Bruschke VG, Sones FM, Jr. Clinical course of patients with normal or slightly or moderately abnormal coronary arteriograms: 10-year follow-up of 521 patients. Circulation. 1980;62(4):712-717.

3. Stone GW, Maehara A, Lansky AJ, de Bruyne B, Cristea E, Mintz GS, Mehran R, et al. A prospective naturalhistory study of coronary atherosclerosis. N Engl J Med. 2011;364(3):226-235.

4. Maddox TM, Stanislawski MA, Grunwald GK, Bradley SM, Ho PM, Tsai TT, Patel MR, et al. Nonobstructive coronary artery disease and risk of myocardial infarction. JAMA. 2014;312(17):1754-1763.

5. Chow BJ, Small G, Yam Y, Chen L, McPherson R, Achenbach S, Al-Mallah M, et al. Prognostic and therapeutic implications of statin and aspirin therapy in individu- 
als with nonobstructive coronary artery disease: results from the CONFIRM (COronary CT Angiography EvaluatioN For Clinical Outcomes: An InteRnational Multicenter registry) registry. Arterioscler Thromb Vasc Biol. 2015;35(4):981-989.

6. Montalescot G, Sechtem U, Achenbach S, Andreotti F, Arden C, Budaj A, Bugiardini R, et al. 2013 ESC guidelines on the management of stable coronary artery disease: the
Task Force on the management of stable coronary artery disease of the European Society of Cardiology. Eur Heart J. 2013;34(38):2949-3003.

7. Johri AM, Behl P, Hetu MF, Haqqi M, Ewart P, Day AG, Parfrey B, et al. Carotid Ultrasound Maximum Plaque Height-A Sensitive Imaging Biomarker for the Assessment of Significant Coronary Artery Disease. Echocardiography. 2016;33(2):281-289. 